\title{
New GPR System Integration with Augmented Reality Based Positioning
}

\author{
Mauricio Pereira \\ School of Engineering, University of \\ Vermont \\ Burlington, Vermont \\ mauricio.pereira@uvm.edu \\ Robert Farrel \\ School of Engineering, University of \\ Vermont \\ Burlington, Vermont \\ bobby.farrel@uvm.edu
}

\author{
Dylan Burns \\ School of Engineering, University of \\ Vermont \\ Burlington, Vermont \\ dylanburns802@gmail.com \\ Dryver Huston \\ School of Engineering, University of \\ Vermont \\ Burlington, Vermont \\ dhuston@uvm.edu
}

\author{
Daniel Orfeo \\ School of Engineering, University of \\ Vermont \\ Burlington, Vermont \\ dan.orfeo@gmail.com \\ Tian Xia \\ School of Engineering, University of \\ Vermont \\ Burlington, Vermont \\ txia@uvm.edu
}

\begin{abstract}
The development of modern cities heavily relies on the availability and quality of underground utilities that provide drinking water, sewage, electric power, and telecommunication services to sustain its growing population. However, the information of localization and condition of subterranean infrastructures is generally not readily available, especially in areas with congested pipes, which impacts urban development, as poorly documented pipes may be hit during construction, affecting services and causing costly delays. Furthermore, aging components are prone to failure and may lead to resources waste or the interruption of services. Ground penetrating radar (GPR) is a promising remote sensing technique that has been recently used for mapping and assessment of underground infrastructure. However, current commercial GPR survey systems are designed with wheel-encoders or GPS for positioning. Wheel-encoder based GPR surveys are restrained to linear-route only, preventing the use of GPR for accurate localization of city wide underground infrastructure inspection. While GPS signal is degraded in urban canyons and unavailable in city tunnels. In this work, we present a new GPR system integration with augmented reality (AR) based positioning that can overcome the limitations of current GPR systems to enable arbitrary-route scanning with a high fidelity. It has the potential for automation of GPR survey and integration with AR smartphone applications that could be used for better planning in urban development.
\end{abstract}

\section{CCS CONCEPTS}

- Hardware $\rightarrow$ Sensor applications and deployments; Sensor devices and platforms;

Permission to make digital or hard copies of all or part of this work for personal or classroom use is granted without fee provided that copies are not made or distributed for profit or commercial advantage and that copies bear this notice and the full citation on the first page. Copyrights for components of this work owned by others than ACM must be honored. Abstracting with credit is permitted. To copy otherwise, or republish, to post on servers or to redistribute to lists, requires prior specific permission and/or a fee. Request permissions from permissions@acm.org.

GLSVLSI'18, May 23-25, 2018, Chicago, IL, USA

(c) 2018 Association for Computing Machinery.

ACM ISBN 978-1-4503-5724-1/18/05 ...\$15.00

https://doi.org/10.1145/3194554.3194623

\section{KEYWORDS}

ground penetrating radar; smart cities; augmented reality; visualinertial odometry; underground infrastructure

ACM Reference Format:

Mauricio Pereira, Dylan Burns, Daniel Orfeo, Robert Farrel, Dryver Huston, and Tian Xia. 2018. New GPR System Integration with Augmented Reality Based Positioning. In GLSVLSI'18:2018 Great Lakes Symposium on VLSI, May 23-25, 2018 Chicago, IL, USA. ACM, New York, NY, USA, 6 pages. https://doi.org/10.1145/3194554.3194623

\section{INTRODUCTION}

The development of modern cities relies heavily on underground utilities that provide drinking water, electric power, sewage, telecommunications, among other services. However the information of the localization and condition of underground infrastructures is generally not readily available, impacting urban development and maintenance. For aging infrastructures, the degraded conditions and defects may result in resources waste and potential service outage. Recently, smart city approaches have been explored to address the issue of city wide mapping and assessing of underground infrastructure [1]. GPR is a remote non-destructive sensing technique $[16,17]$ that has been used to both image underground infrastructure and assess buried pipe condition $[5,7,8,15]$. However, for city wide mapping and assessment of underground infrastructure, an accurate positioning system is required. Current commercial GPR are based on wheel-encoders for local positioning and GPS for global positioning. Wheel-encoders provide linear accuracy only, implying that surveys have to be performed in straight-line routes for correct localization of underground features. While GPS provides flexibility, its resolution is rough for precisely positioning subsurface objects. Moreover GPS signals may be degraded in urban canyons, or unavailable in certain areas, i.e. in tunnels. These factors result in significant limitations for employing existing commercial GPRs for city wide survey applications, especially in dense urban areas where underground infrastructure is critical. Hence, a versatile, accurate, low-cost positioning solution is required to fully explore GPR potentials for underground infrastructure mapping and assessment. 


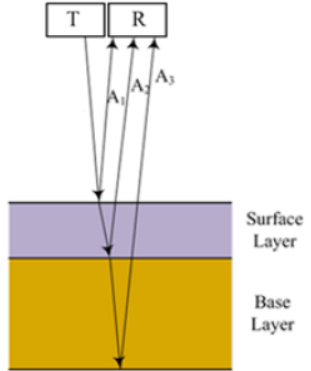

Figure 1: Scheme of a typical GPR system [14].

Recently, there has been great technology advancement in augmented reality (AR) applications for smartphones. For instance, Google created a framework named Tango for the development of AR applications [2]. This framework leverages on photogrammetry techniques that, combined with smartphone's IMU data, enable accurate local positioning.

In this work, a Tango enabled Lenovo Phab 2 Pro smartphone, here denominated the Tango device, was integrated with a GSSI SIR 30 GPR system using an Arduino microcontroller equipped with an XBee bluetooth (BT) shield and a relay for GPR scan triggering. A smartphone application using the Tango framework was developed with Unity, a game development platform that supports mobile AR applications [4]. The application controls communication with the BT shield, sends GPR scan triggering messages to the Arduino and logs device position. This integrated system enables GPR scanning in an arbitrary route with good positioning accuracy, expanding GPR survey capabilities.

This paper is organized as follows, section 2 describes the fundamentals of GPR sensing, section 3 introduces the Tango framework features, section 4 explains the system integration and operation, section 5 shows results demonstrating that this new GPR system is capable of performing arbitrary-route GPR survey, and section 6 presents the concluding remarks.

\section{GROUND PENETRATING RADAR}

GPR is a non-destructive evaluation technique that has been traditionally used to image the subsurface structure or detect the burying objects. Recently it has been applied to the mapping and assessment of underground infrastructure [8, 9]. A typical GPR consists of a transmitting antenna that sends an electromagnetic wave into the subsurface and a receiving antenna that collects scattered signals as illustrated in Fig. 1. Signals are scattered at every change in the dielectric property of the media, such that GPR can detect both metallic and non-metallic objects [10].

The corresponding received signal, denominated an A-scan, is shown in figure 2. The $A_{0}$ peak is the direct coupling between transmitter and receiver, $A_{1}$ is the ground reflection, $A_{2}$ and $A_{3}$ are signals due to the difference in layers dielectric properties. In a GPR survey, signal $A_{2}$ would be produced by the presence of buried pipes or other subterranean infrastructure.

Current GPR surveys are conducted over a straight-line route, collecting several A-scans to compose a B-scan. B-scans are represented as a grayscale image whose intensity is proportional to

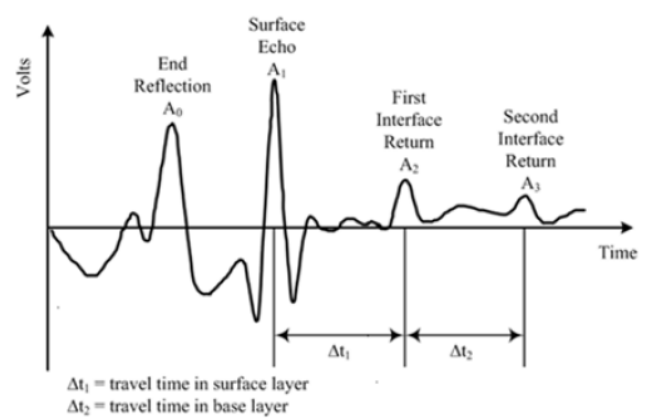

Figure 2: Example of a GPR A-scan signal [14].

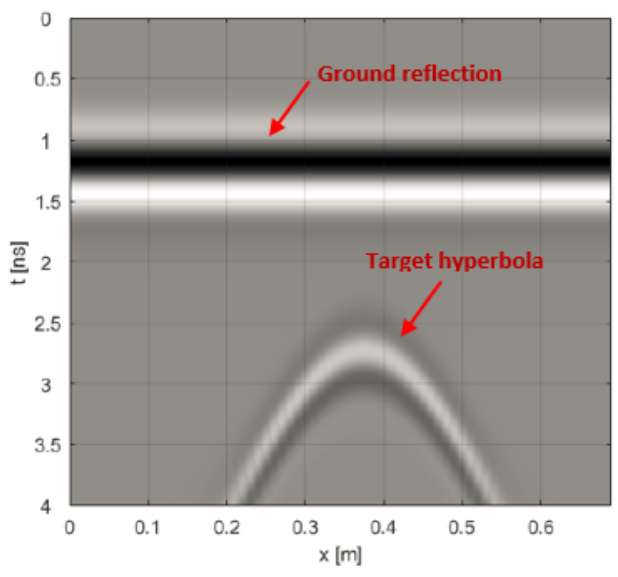

Figure 3: Example of a GPR B-scan. Each pixel column corresponds to an A-scan.

the received signal. For a target buried in the ground, as the GPR system scans, the time index of the scattering corresponding to the target varies, and achieves the lowest value when the GPR is right over the target which is reflected in B-scans as hyperbolas, illustrated in Fig. 3.

By evaluating a B-scan image, it is possible to localize an underground feature and infer subsurface structure conditions [6, 7]. However, for mapping purposes, the A-scans must be associated to a position in the survey area.

In wheel-encoder based GPR systems, each A-scan collection is triggered off of the wheel-encoder signal and each A-scan can be correlated to a linear position. However, the wheel-encoder does not take into consideration variations in height or deviations of the straight-line route. As a result, the subsurface features can be incorrectly positioned. In challenging terrains, wheel-encoder based GPR survey may become unfeasible. Furthermore, for a complete mapping of the area, multiple B-scans have to be collected and composed to form a C-scan, which is a tedious and slow process both for collection and post processing as the operator must carefully keep track of the relative position among B-scans. 


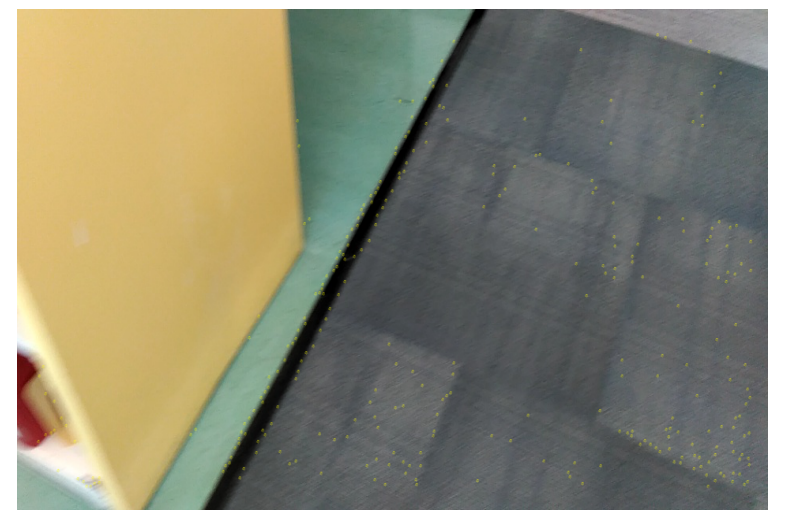

Figure 4: Tango feature points (yellow dots) detected using its fish-eye and RGB cameras.

\section{TANGO}

Recent advances in smartphone technology have endowed these devices with an array of sensors beyond the traditional RGB camera, such as a wide-angle fish-eye camera and time-of-flight camera that enabled the use of advanced visual-inertial odometry (VIO) algorithms for AR applications. Google Tango project provides a VIO framework for smartphones and features mapping capability to mitigate IMU drifts through loop closures. A Tango device provides long-term position accuracy and has been used to map and reconstruct large-scale areas [13]. Furthermore, the framework is suited for integration with AR technology of interest for smart city applications $[11,12]$.

\subsection{Tango Framework}

Tango is a framework for the development of AR applications that explores VIO for localization and mapping [2]. Localization and mapping in Tango are based on three core features: motion tracking, where IMU and camera data are fused to provide accurate position estimate; local area mapping, where key visual features of the environment are stored and later used for device relocalization; depth sensing, where a 3D understanding of the environment is achieved based on time-of-flight camera. For our GPR integration, the main features of interest are motion tracking, and local area mapping and device relocalization. The latter is described in more detail in the next section.

\subsection{Local Area Mapping and Relocalization}

Tango creates local area maps based on features in the images captured by its fish-eye camera and RGB camera. During mapping, Tango applications track distinguished visual features of the environment such as edges, corners and other visual cues [2]. Figure 4 shows an example of visual features tracked by the RTAB-Map Tango application [3]. Maps hold key features of the environment in a compact indexed form such that map querying is performed in real time and the device can be quickly relocalized. Relocalization, or loop closure, is achieved by matching features in the current view of the device camera to those stored in the map. Through loop closure, IMU drifting can be mitigated.

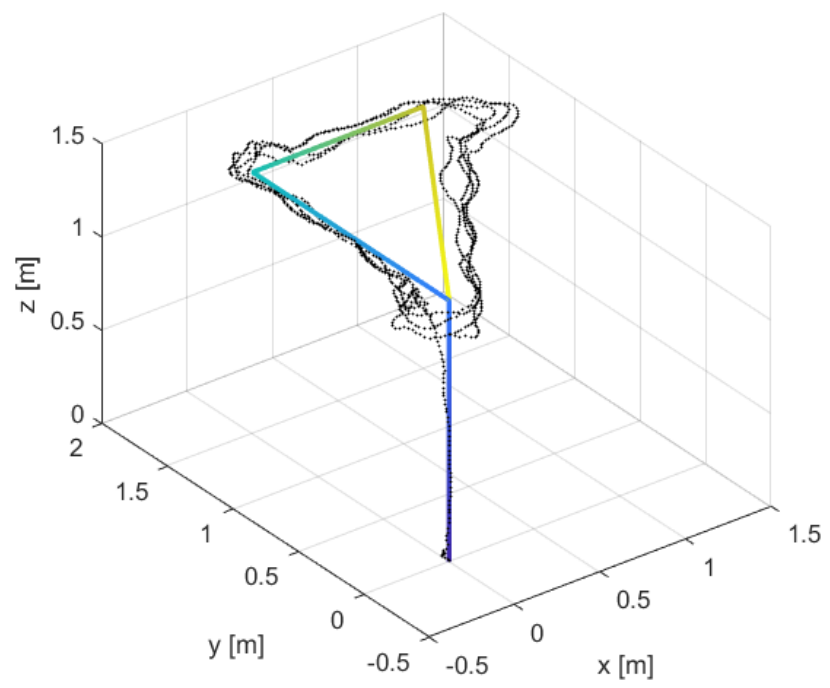

Figure 5: Position tracked by Tango (dots) and ground truth (gradient line). Tango accurately captures height and horizontal displacement in all directions.

\subsection{Tango Device Evaluation}

To illustrate Tango positioning capability, tracking of a small scale indoor loop route was performed. The setup is as follows, the device is started at ground level, and height is also tracked by Tango. The device is brought at a height of $1.4 \mathrm{~m}$, moved $1.5 \mathrm{~m}$ ahead, turned right and moved $1 \mathrm{~m}$ in that direction. Finally, the device is brought back to the starting position. This route was looped through 4 times to evaluate Tango accuracy. Fig. 5 shows the assumed ground truth, depicted by the gradient colored line, and the position as tracked by Tango. Notice that all loops are close to each other and in good agreement with the ground truth, as well as the indicated height. This demonstrates that Tango has good accuracy, on sub-meter range, in all directions for an indoor, static environment case.

However, Tango accuracy is affected by several factors, for instance, change in lighting conditions, or an excessively dynamic environment, as well as potential drifts when area learning was not performed. To illustrate Tango performance in an outdoor, dynamic environment, an experiment tracking a long-distance walk $(>50 \mathrm{~m})$ on the University of Vermont campus was performed. In this experiment, a measured ground truth was not available. Instead we evaluate how well would the local positions match the traveled path when transformed to a geographic coordinate, by overlay on satellite imagery. In the experiment, only the motion tracking feature was used, hence drift mitigation by loop closure was not present. The smartphone Tango application program was set to $\log$ position by every 1 inch displacement. The local position is given in relation to the device starting pose. By keeping track of the initial position and orientation in relation to a geographic coordinate system, it is possible to obtain global coordinates by adding the local map recorded. In this experiment, we used the Esri ArcGIS software suite to add the recorded local map to a global map. In this experiment, a footpath at the University of Vermont campus is followed, and involves the following challenges: long distance 


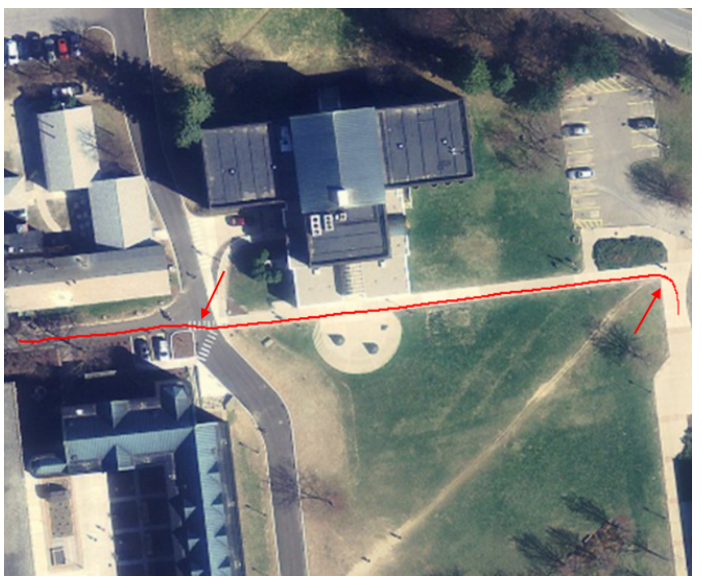

Figure 6: Position tracked by Tango overlaid on satellite imagery corresponding to its global position. Notice that the recorded position stays within the footpath. Arrows indicate both a subtle change in the linear-route (left) and a curve (right) that are not detectable with current wheel-encoder based GPR.

measurement, dynamic environment, and curves. The tracked path is shown in Fig. 6. The walk started on the left side of the indicated route. Notice that Tango accurately kept track of the distance covered, closely matching the footpath in spite of not using the loop closure feature, indicating meter-range accuracy or better. Furthermore, changes in relation to a perfect linear-route are indicated, in particular a subtle change at a crosswalk and a 90 degrees turn at the end of the walk. Such nonlinear route change can not be tracked by wheel-encoders in GPR systems. Nevertheless our GPR system is capable of pinpoint the position for each A-scan wave collected.

\section{SYSTEM INTEGRATION AND OPERATION}

The integrated system is composed of the Tango device, the Arduino microcontroller with both the BT shield and relay, and the GSSI SIR-30 radar system (see Fig. 7).

The GSSI GPR includes a $400 \mathrm{MHz}$ antenna and a SIR-30 control unit. The Tango device is mounted in front of the cart and communicates with the Arduino microcontroller via BT. The Arduino and relays are connected to the SIR-30 and control scan triggering.

Before GPR sensing, a visual mapping of the area is performed using the Google Tango Explorer application program. In the GPR sensing phase, the previously recorded map is loaded by our smartphone application. Once relocalization occurs, the Tango framework can be used to retrieve the device position and attitude. Position is described in terms of cartesian coordinates in relation to the survey area map reference frame, while attitude is given by a quaternion describing the rotation in relation to that reference frame.

A high-level diagram of the integrated system is presented in Fig. 8. The Tango device sends a trigger signal to the Arduino microcontroller should its spatial position changes by a predefined threshold. While the threshold of the Tango device spatial offset can

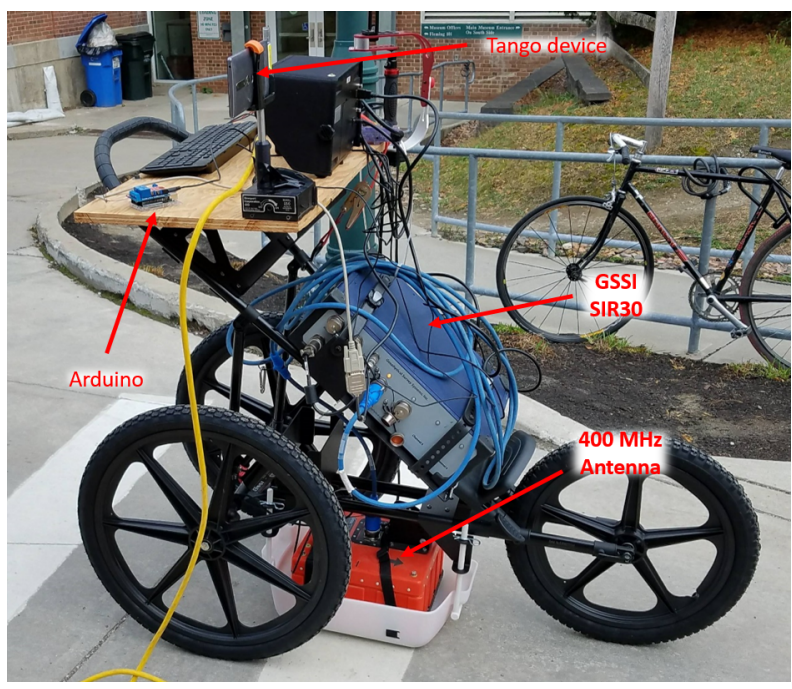

Figure 7: A GPR cart with the GSSI SIR-30 control unit, a 400MHz antenna, the Tango device and the Arduino board with the BT shield and relays.

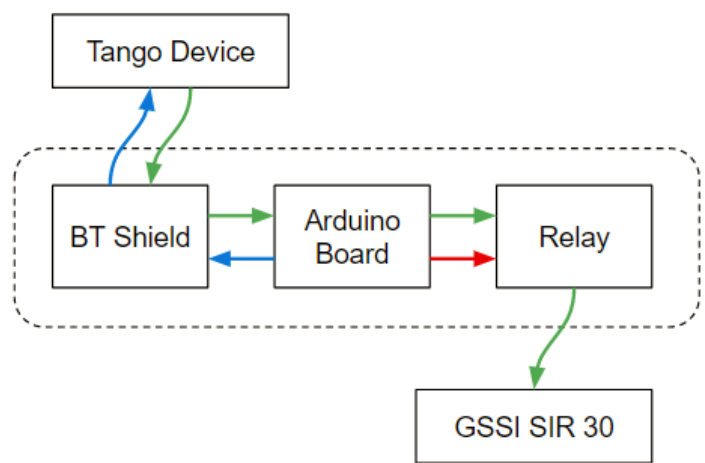

Figure 8: Diagram of the system integration.

be set to an arbitrary value, our operation experience suggests that for mid to long range scans, setting 1 inch threshold allows Tango framework to reach optimum ranging performance. Therefore in our GPR system integration, 1 inch threshold value is adopted.

Correspondingly the Arduino controls the relay to trigger GPR scan operation. As Arduion microcontroller receives the Tango message, it turns on the relay to trigger GPR to perform A-scans. Relay contact time is set to an interval of $25 \mathrm{~ms}$ compromising between acquisition frequency and preventing relay excessive stress. With the relay adopted, its minimum callback time can be set to $5 \mathrm{~ms}$. An alternative option is to use transistors with nano second triggering time to replace the relay. After the relay is turned off, the Arduino microcontroller sends a callback signal to the smartphone informing that it is ready to receive another triggering signal. Radar triggering and location registration are performed simultaneously, such that each A-scan can be matched to a position in the reference map such tha the detected features can be correctly positioned. 


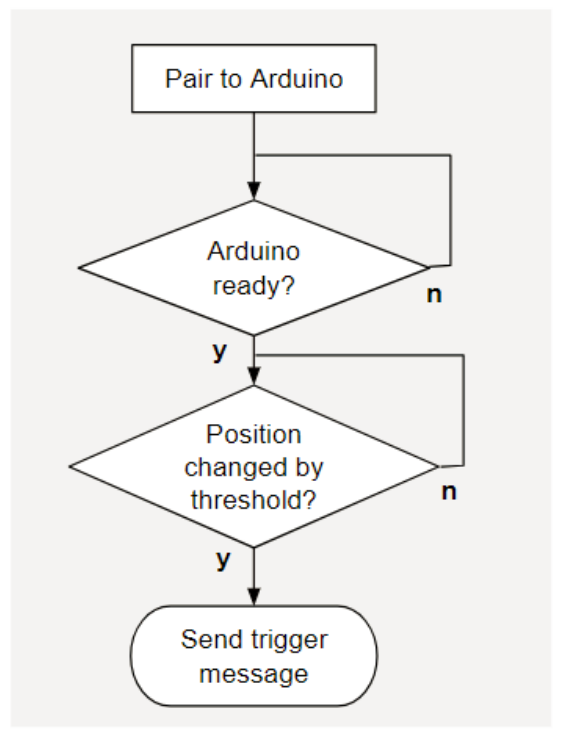

Figure 9: Application logic on the Tango device side.

\subsection{Bluetooth Controller}

To establish the BT communication, the TechTweaking Bluetooth adapter library for Unity was used. The library offers BT pairing capability, and sending and receiving streaming functions.

4.1.1 Tango device side. The diagram shown in Fig. 9 depicts the logic flow on the Tango application. First the Tango device and the Arduino BT shield must be paired to exchange messages. Once paired, an internal variable on the Tango device keeps track of whether the Arduino is ready to receive another trigger signal. If the Arduino is ready, a change in position check is performed. If the distance threshold is achieved, a trigger message is sent to the Arduino and the current device position is logged. These checks are performed at every application frame rendering. When the Arduino relay is opened, the Arduino will respond with a message informing that it is ready to receive another trigger message. Arduino readiness is determined on the Arduino side as a function of the relay status.

4.1.2 Arduino side. The diagram in Fig. 10 shows Arduino logic flow. As Tango device and the XBee BT shield are paired, a communication channel is established, and a stream reader can be used to receive messages sent by the Tango device. If a trigger message is received, the relay will be closed for $25 \mathrm{~ms}$, activating the A-scan collection by the GSSI SIR-30. Upon release, the Arduino uses the stream channel to send a callback message to the smartphone, signaling the release of the relay.

\section{RESULTS}

A circular long-range GPR scan was performed at the University of Tennessee, Chattanooga, using the new GPR system integrated with the Tango device. The 3D imaging of the subsurface is depicted in Fig. 11a in local coordinates. This local area map can be anchored to a world coordinate of choice, and have the detected features

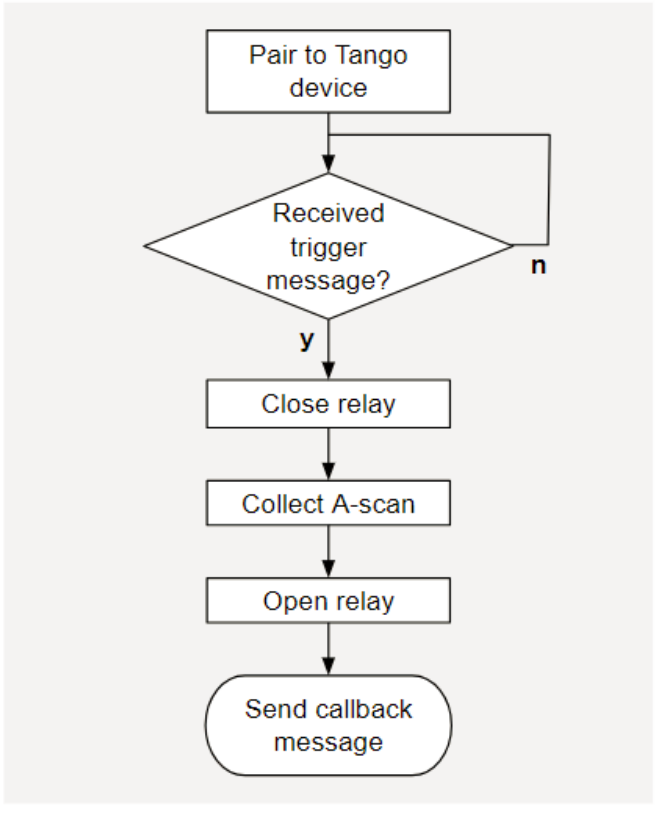

Figure 10: Application logic on the Arduino side.

positioned in a geographic coordinate system. To perform this last step, the starting position and orientation of the survey in relation to a geographic coordinate system must be known, in particular its orientation in relation to the north and its starting latitude and longitude. In this survey, we used Esri ArcGIS to add our local recorded positions to a geographic coordinate system. The overlay of the tracked path and satellite imagery is shown in Fig. 12 as a yellow curve. The recorded position successfully matches the surveyed area.

As an example of positioning of detected targets, consider the detail of the 3D image highlighting a detected underground feature, possibly a pipe connected to an adjacent sink, in Fig. 11b. The position of this target is assumed to be at the top of the hyperbola, and both the local position and the corresponding latitude and longitude are shown. In Fig. 12 we indicate the corresponding global position of this feature as a red dot. It corresponds to the expected position, adjacent to one of the sinks present in the area, thus successfully correlating a feature detected in a nonlinear GPR scan to a geographic coordinate.

This survey demonstrates that our new GPR system is capable of performing nonlinear GPR scans with good position accuracy and that recorded positions can be readily associated to geographic coordinates. This opens venue for the automation of GPR survey since scans can be collected continuously as well as potential integration with AR technology applications.

\section{CONCLUSIONS}

In this paper a new GPR system integration with AR based positioning available on smartphones was introduced. The fundamentals of GPR sensing and the Google Tango AR technology were presented. 


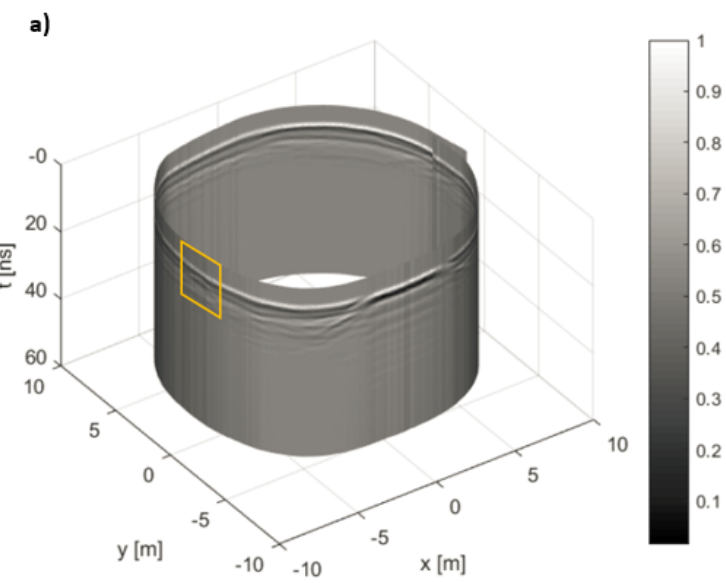

b)



Figure 11: (a) 3D subsurface imaging of the surveyed area in local coordinates and (b) Detail of an underground detected feature showing both its local and global coordinates.

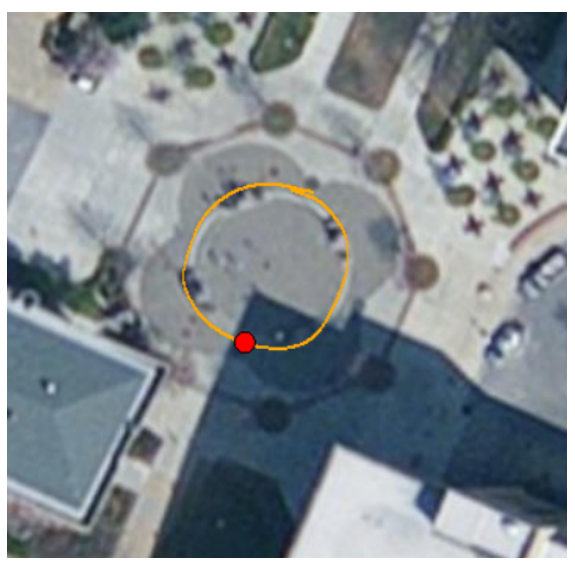

Figure 12: Top view of the survey area with circular path tracked by Tango. Highlighted red point indicates the position of the feature detailed above.
This new GPR system is capable of performing arbitrary route scanning, challenging the GPR industry paradigm of linear-route scanning only. The system capability was demonstrated by successfully performing a circular GPR scan over a distance of $50 \mathrm{~m}$ and by assigning a geographic coordinate to detected features.

\section{ACKNOWLEDGMENTS}

This work is supported by the NSF Grants No. 1647095 and No. 1640687, the UVM SparkVT Fund, and VT EPSCoR.

\section{REFERENCES}

[1] 2017. Chicago maps its underground maze. (2017). https://www.cnet.com/news/ chicago-maps-its-underground-maze-rahm-emanuel/

[2] 2018. https://developers.google.com/tango/. (2018). https://developers.google. com/tango/

[3] 2018. RTAB-Map. (2018). http://introlab.github.io/rtabmap/

[4] 2018. Unity Mobile AR. (2018). https://unity3d.com/solutions/mobile-ar

[5] Amr Ahmed, Yu Zhang, Dylan Burns, Dryver Huston, and Tian Xia. 2016. Design of UWB antenna for air-coupled impulse ground-penetrating radar. IEEE Geoscience and Remote Sensing Letters 13, 1 (2016), 92-96.

[6] L. Capineri, P. Grande, and J. A.G. Temple. 1998. Advanced image-processing technique for real-time interpretation of ground-penetrating radar images. International fournal of Imaging Systems and Technology 9, 1 (1998), 51-59. https://doi.org/10.1002/(SICI)1098-1098(1998)9:1<51::AID-IMA7>3.0.CO;2-Q

[7] Sevket Demirci, Enes Yigit, Ismail H. Eskidemir, and Caner Ozdemir. 2012. Ground penetrating radar imaging of water leaks from buried pipes based on backprojection method. NDT and E International 47 (2012), 35-42. https://doi.org/10. 1016/j.ndteint.2011.12.008

[8] Dryver Huston, Tian Xia, Yu Zhang, Taian Fan, Dan Orfeo, and Jonathan Razinger. 2017. Urban underground infrastructure mapping and assessment. April (2017), 101680M. https://doi.org/10.1117/12.2263530

[9] Dryver Huston, Tian Xia, Yu Zhang, Taian Fan, Dan Orfeo, and Jonathan Razinger. 2017. Urban underground infrastructure mapping and assessment. In Sensors and Smart Structures Technologies for Civil, Mechanical, and Aerospace Systems 2017, Vol. 10168. International Society for Optics and Photonics, 101680M.

[10] Harry M. Jol. 2009. Ground penetrating radar: theory and applications (1 ed.). Elsevier Science, Amsterdam, Netherlands.

[11] Boris Pokric, Srdan Krco, and Maja Pokric. 2014. Augmented Reality Based Smart City Services Using Secure IoT Infrastructure. 2014 28th International Conference on Advanced Information Networking and Applications Workshops (2014), 803-808. https://doi.org/10.1109/WAINA.2014.127

[12] Gerhard Schall, Erick Mendez, Ernst Kruijff, Eduardo Veas, Sebastian Junghanns, Bernhard Reitinger, and Dieter Schmalstieg. 2009. Handheld Augmented Reality for underground infrastructure visualization. Personal and Ubiquitous Computing 13, 4 (2009), 281-291. https://doi.org/10.1007/s00779-008-0204-5

[13] Thomas Schneider, Marcin Dymczyk, Marius Fehr, Kevin Egger, Simon Lynen, Igor Gilitschenski, and Roland Siegwart. 2017. maplab: An Open Framework for Research in Visual-inertial Mapping and Localization. (2017). https://doi.org/10. 1109/LRA.2018.2800113 arXiv:1711.10250

[14] Anbu Selvam Venkatachalam. 2013. RF Circuit Development for Ultra-wideband Ground Penetrating Radar Evaluating Highway Pavement and Bridge Deck Surface. Master's thesis. University of Vermont, Burlington, VT.

[15] Tian Xia, Xianlei Xu, Anbu Vekatachalam, and Dryver Huston. 2012. Development of a High Speed UWB GPR for Rebar Detection. In 14th International Conference on Ground Penetrating Radar (GPR).

[16] Yu Zhang, Anbu Selvam Venkatachalam, Dryver Huston, and Tian Xia. 2014. Advanced signal processing method for ground penetrating radar feature detection and enhancement. In Nondestructive Characterization for Composite Materials, Aerospace Engineering, Civil Infrastructure, and Homeland Security 2014, Vol. 9063. International Society for Optics and Photonics, 906318.

[17] Yu Zhang, Anbu S Venkatachalam, and Tian Xia. 2015. Ground-penetrating radar railroad ballast inspection with an unsupervised algorithm to boost the region of interest detection efficiency. Journal of Applied Remote Sensing 9, 1 (2015), 095058. 